\title{
ROBÓTICA EDUCATIVA EN CONTEXTOS INCLUSIVOS: EL CASO DE LAS AULAS HOSPITALARIAS
}

\section{(EDUCATIONAL ROBOTICS IN INCLUSIVE CONTEXTS: THE CASE OF THE HOSPITAL CLASSROOMS)}

Carina S. González-González

Universidad de La Laguna

Verónica Violant Holz

Universidad de Barcelona

Alfonso Infante Moro

Universidad de Huelva

Lorena Cáceres García

Universidad de Barcelona

María Dolores Guzmán Franco

Universidad de Huelva

DOI: 10.5944/educXX1.27047

\section{Cómo referenciar este artículo/How to reference this article:}

González-González, C.; Violant Holz, V.; Infante Moro, A; Cáceres García, L. y Guzmán Franco, M.D. (2021). Robótica educativa en contextos inclusivos: el caso de las aulas hospitalarias. Educación XX1, 24(1), 375-403, http://doi.org/10.5944/educXX1.27047

González-González, C.; Violant Holz, V.; Infante Moro, A.; Cáceres García, L. \& Guzmán Franco, M.D. (2021). Educational robotics in inclusive contexts: The case of the hospital classrooms. Educación XX1, 24(1), 375-403, http://doi.org/10.5944/educXX1.27047

\section{RESUMEN}

Los derechos fundamentales de los menores hospitalizados incluyen la necesidad de ofrecer la atención educativa comprendiendo los aspectos emocionales durante la situación de enfermedad y convalecencia. La garantía de estos derechos, desde una mirada en pedagogía hospitalaria, acompañado de profesionales formados, facilita las buenas prácticas. Actualmente la enseñanza del pensamiento computacional y la programación constituyen una nueva alfabetización que debe ser llevada a todas las aulas, incluidas las aulas hospitalarias. Alfabetización que debería contener el aprendizaje de la regulación emocional. En este trabajo se presenta un estudio de caso sobre una intervención educativa inclusiva y adaptable a las características de las aulas hospitalarias para la enseñanza del pensamiento 
computacional de forma transversal y la programación sin pantallas y con robots, teniendo como uno de los elementos importante de desarrollo de la competencia emocional. Los resultados indican que la intervención mejora el estado emocional de los menores, así como sus conocimientos respecto al pensamiento computacional.

\section{PALABRAS CLAVE}

Emoción, pensamiento computacional, escuela en hospitales, pedagogía diferencial, pedagogía hospitalaria

\section{ABSTRACT}

The fundamental rights of hospitalized minors include the need to offer educational care, including emotional aspects during the situation of illness and convalescence. The guarantee of these rights from a perspective of hospital pedagogy accompanied by specialized professionals facilitates good practices. Currently the teaching of computational thinking and programming constitutes a new literacy that must be carried to all classrooms, including hospital classrooms. Literacy should involve learning about emotional regulation.

This paper presents a case study on an educational intervention that is inclusive and adaptable to the characteristics of hospital classrooms for the teaching of computational thinking in a transversal way and programming without screens and robots, having the development of emotional competence as one of the important elements. The results indicate that the intervention improves the emotional state of the children, as well as their knowledge regarding computational thinking.

\section{KEYWORDS}

Emotion, computational thinking, hospital teaching, differentiated teaching, hospital pedagogy

\section{INTRODUCCIÓN}

En la sociedad actual, el proceso de socialización cultural es predominantemente digital. La población se encuentra inmersa en un ecosistema de información multimodal donde la tecnología forma parte 
CARINA S. GONZÁLEZ-GONZÁLEZ, VERÓNICA VIOLANT HOLZ, ALFONSO INFANTE MORO,

de la vida cotidiana y donde las experiencias, incluidas las de enseñanzaaprendizaje, se encuentran mediadas por la tecnología de la información y comunicación (Area, 2015; Aretio, 2019; Dufva \& Dufva, 2019). Asimismo, los medios tecnológicos se hacen presentes también en los contextos hospitalarios, abriendo numerosas posibilidades hacia la normalización de las personas en situación de enfermedad y convalecencia (Serrano, 2013).

No cabe duda de que la tecnología es uno de los factores que pueden favorecer los procesos de integración, inclusión y normalización, permitiendo crear espacios de atención educativa virtuales (Ocampo y Lizasoáin, 2019), así como expandir, integrar y potenciar los espacios presenciales con metodologías flexibles, adaptadas a las necesidades del alumnado hospitalizado (González-González y Violant, 2015; Morales, 2019). Existe también, un amplio abanico de herramientas y aplicaciones informáticas que pueden ser usadas en los contextos hospitalarios (González-González y Violant, 2019; Ryu et al., 2013; Trost, 2019). Ejemplo que ello son las aplicaciones para trabajar competencias digitales y de salud de los niños, niñas y jóvenes (Maher, 2020), que permiten inventar y crear tecnología desde la competencia STEAM en las aulas hospitalarias, a través de herramientas de trabajo colaborativo, en redes sociales y videojuegos y, ampliando el uso social y lúdico-educativo de las tecnologías (GonzálezGonzález et al., 2019) y no solo consumirla. En este sentido, se puede utilizar la robótica en este contexto para favorecer la adquisición de competencias, tales como la emocional y la digital (González-González et al., 2019). El uso de la robótica en contextos hospitalarios es un recurso valioso que puede ser aprovechado para apoyar el desarrollo de las actividades del aula hospitalaria (Prendes, 2014) que demuestra beneficios positivos de su utilización, así como una buena aceptación por parte de los niños y niñas, sus familias y los profesionales de la salud y educadores (Dawe et al., 2019).

El uso de la tecnología, en el AH, debe acompañase de la alfabetización digital, la enseñanza del pensamiento computacional y la programación introducida en los sistemas educativos (Balanskat \& Engelhardt, 2015; Bocconi et al., 2016; García-Peñalvo, 2017; Llorens et al., 2017; Ministerio de Educación, Cultura y Deportes, 2018; Pretz, 2014; Resnik, 2013) con ciudadanos que aprendan a "codificar" para ser productores digitales de información, no solo consumidores digitales de la misma (Bers, 2008). El AH, como espacio ubicado en el hospital, tiene como objetivo brindar atención escolar al alumnado en situación de enfermedad en edades comprendidas entre la etapa infantil y la secundaria (Violant et al., 2009). En el AH se busca también normalizar la vida de los menores hospitalizados, ayudarles a comprender los procesos de su enfermedad. Estudios han demostrado la efectividad de introducir programas de preparación psicopedagógica para reducir y prevenir la aparición de síntomas de ansiedad en pacientes 
pediátricos hospitalizados (Lizasoáin \& Polaino, 1995) y los déficits en el autoconcepto (Polaino-Lorente y Lizasoáin, 1994). Por otra parte, también se les ayuda conectando el hospital con la escuela durante el proceso de hospitalización y ayudándoles a reincorporarse cuando finaliza el mismo (González-González et al., 2011). Dicho espacio es garantizado como recurso público en España, en base al acuerdo en 1998, entre el ministerio de Educación y Cultura, el Ministerio de Sanidad y Consumo y el Instituto Nacional de Salud y toma el perfil de la escuela unitaria (Guillén y Mejia, 2002). Por las condiciones especiales de las aulas hospitalarias, la metodología de trabajo debe presentarse de manera flexible y activa; permitir el aprendizaje basado en proyectos que partan de los intereses y adaptado a las características particulares del alumnado, y fomentar la creatividad, las emociones, la socialización, así como el tener un carácter lúdico-educativo (Antequera \& Domíguez, 2015, Barquero, 2017; GonzálezGonzález y Violant, 2015). En el anexo 1 se muestran proyectos llevados a cabo en aulas hospitalaria (AH) de España, con la utilización de algunos de robots para estos fines.

El entorno hospitalario supone la inesperada irrupción de esta en la vida de la persona, la posiciona en una situación de debilidad (HernándezPérez y Rabadán, 2014), puede resultar desmotivador y provocar emociones negativas como miedo y tristeza, afectando y condicionando su rendimiento escolar, así como a su salud (Castellanos y Torres, 2015; De MulaFuentes et al., 2018; Naranjo y Castillo, 2006; Ortigosa et al., 2000). Por ello, es importante inducir estados emocionales positivos que favorezcan su motivación durante su estancia en el hospital, y la robótica educativa junto a estrategias basadas en juegos-actividad lúdica, han demostrado ser herramientas efectivas (Antequera et al., 2015; Nuncira et al., 2019; Romero y Alonso, 2007; Sequeira et al., 2019; Serrano, 2013, 2019; Smith et al., 2020).

Este hecho requiriere de profesionales formados (Violant y Molina, 2010) y específicamente formados en competencias digitales para que los aprendizajes puedan realmente ser significativos (González-González, 2015; Prendes, 2014; Violant, 2015), pero también en competencias emocionales con el fin de dar respuesta al impacto que genera la situación de enfermedad y convalecencia desde la atención educativa y lúdica (Bisquerra, 2015; Lizasoáin y Violant, 2015; Violant et al., 2009). Hen (2020) nos remarca, desde los resultados de su estudio sobre la formación de los maestros hospitalarios en inteligencia emocional, que esta formación debe ir acompañadas de trabajo de campo y procesamiento significativo, y no únicamente de elementos teóricos. 
CARINA S. GONZÁLEZ-GONZÁLEZ, VERÓNICA VIOLANT HOLZ, ALFONSO INFANTE MORO,

El derecho a la educación en el hospital es crucial y debe ser ofrecido (Artioli, 2018) y garantizado desde el marco de los derechos de la infancia hospitalizada (Asensio-Ramon, 2018) y desde la Pedagogía hospitalaria (Calvo, 2017, Lizasoáin et. al., 2015). La pedagogía hospitalaria es una estrategia que permite hacer frente a la enfermedad y reconocer a los niños y a sus cuidadores como sujetos de derechos y oportunidades, y consolidar el trabajo intersectorial e interdisciplinario para el desarrollo de la salud del niño (Valencia et al., 2019, p.1).

Los autores a lo largo del artículo responderán a dos preguntas: 1/ ¿Es posible introducir la enseñanza del pensamiento computacional de forma transversal a través de la robótica educativa en las aulas hospitalarias?; 2/ $¿$ Existen diferencias significativas entre las emociones de entrada y salida después de la intervención con el kit de robótica educativa KIBO en el aula hospitalaria $(\mathrm{AH})$ ?

En este trabajo nos centraremos en estudiar el impacto emocional de la introducción de la robótica educativa en las AH, en particular, nos centraremos en estudiar un caso concreto que es el aula hospitalaria de un hospital de Canarias.

\section{MÉTODO}

\section{Diseño}

El presente trabajo presenta como caso de estudio una experiencia piloto de introducción de la enseñanza del pensamiento computacional a través de robótica educativa como un recurso didáctico transversal en el aula hospitalaria del Hospital Universitario de Canarias y mide el nivel de impacto emocional que tiene esta estrategia en el aula hospitalaria con niños, niñas y jóvenes que se encontraban hospitalizados en el momento de su aplicación en el aula hospitalaria. A continuación, se describirá el contexto, la muestra, los instrumentos, el análisis y los resultados obtenidos en dicha intervención.

\section{Contexto}

La propuesta metodológica se llevó a cabo en el aula hospitalaria del Hospital Universitario de Canarias (HUC), ubicado en San Cristóbal de la Laguna, Tenerife (Canarias). El HUC es un centro público dependiente de la Consejería de Sanidad del Gobierno de Canarias. Es un hospital de tercer nivel orientado a la asistencia médica del norte de Tenerife, también 
es el hospital de referencia para las islas menores de la provincia de La Palma, La Gomera y El Hierro. El hospital tiene en la planta de Pediatría un aula hospitalaria "las Andoriñas", destinada a continuar con el desarrollo educativo, afectivo y social de los niños, niñas y jóvenes que se encuentran hospitalizados, siempre y cuando su estado médico lo permita, y siendo uno sus los objetivos principales el de ayudar al estudiante a valorar su enfermedad y a reducir los miedos y ansiedades.

Por otra parte, la atención educativa de los menores que atiendan al aula hospitalaria se realiza en las diferentes etapas correspondientes, tales como Educación Infantil, Enseñanza Básica o Bachillerato (Dirección General de Ordenación, Innovación y Promoción Educativa. Consejería de Educación y Universidades, 2018). Cada curso escolar las aulas hospitalarias de Canarias deben dar apoyo educativo a cientos de menores de diferentes edades. En ingresos inferiores a 10 días se realizan principalmente actividades lúdicoeducativas y en hospitalizaciones prolongadas se realizan programaciones coordinadas con los centros educativos de referencia, trabajando diferentes ámbitos, tales como el educativo, el lúdico o el desarrollo personal. El tiempo de estancia medio es de 7 a 15 días (Ramos et al., 2014).

Cabe destacar que el contexto es altamente variable: nunca se sabe cuántos niños y niñas pueden acudir al aula hospitalaria hasta el momento de comenzar la sesión, se desconocen las edades, que pueden ir de 3 a 16 años y las características particulares de cada caso. Por ello, se recomienda trabajar por proyectos, con una programación flexible y con objetivos a desarrollar y cumplir en una misma sesión.

El docente del aula es nombrado por la Consejería de Educación y normalmente, desempeña solo su labor, con apoyo del voluntariado de diferentes asociaciones y/o estudiantes en práctica.

\section{Participantes}

Los participantes del estudio fueron los niños, niñas y jóvenes que se encontraban hospitalizados y asistieron al aula hospitalaria durante los días en que se llevaron a cabo las cinco sesiones programadas durante los meses de mayo y junio de 2018. La asistencia al aula hospitalaria no es obligatoria y depende del estado de salud del menor.

La muestra se compuso de $\mathrm{N}=22$ participantes (21 niñas y un niño) en edades comprendidas entre los 4 y los 16 años $(M=12.4)$. El lugar de procedencia del alumnado es la zona norte de la isla de Tenerife. Las patologías de los menores son diversas, destacando la prevalencia de 
CARINA S. GONZÁLEZ-GONZÁLEZ, VERÓNICA VIOLANT HOLZ, ALFONSO INFANTE MORO,

adolescentes ingresadas por trastornos en la alimentación. Por tanto, tenemos una muestra heterogénea que comparte las características a estudiar: el contexto y su estado emocional en la situación de hospitalización.

La representatividad de la muestra viene determinada por las características que evaluamos en cada participante relacionadas con el problema de estudio, la forma de medirlas y la información obtenida desde las mismas para la evaluación (Yanow \& Schwartz-Shea, 2015).

Los tutores (padres, madres y responsables legales) de participantes firmaron el consentimiento informado del estudio, y la intervención fue aprobada por el Comité responsable de la Consejería de Educación de Canarias. Asimismo, se informó y se solicitó la autorización para la utilización de imágenes.

\section{Procedimiento}

Para poder llevarla a este tipo de aulas, se diseñó una intervención educativa inclusiva y adaptable a las características de las aulas hospitalarias para la enseñanza del pensamiento computacional y la programación sin pantallas y con robots.

El trabajo se basó en una propuesta metodológica para trabajar el pensamiento computacional mediante un kit de robótica llamado KIBO robot (Bers, 2018). La propuesta metodológica transcurrió en el aula hospitalaria anteriormente citada.

El objetivo principal de las sesiones fue ayudar a los menores a comprender y expresar sus sentimientos y emociones, producidos por la situación de hospitalización que están vivenciando.

Los objetivos de aprendizaje relacionados con el pensamiento computacional que se persiguieron con las actividades fueron:

1. Aprender y aplicar el proceso de ingeniería en la construcción de cosas (por ejemplo, los robots).

2. Aprender los diferentes componentes de un robot y cómo funcionan.

3. Aprender las instrucciones de programación.

4. Adquirir las competencias de pensamiento computacional. 
Las estrategias llevadas a cabo en las diferentes sesiones siguieron una estructura básica:

a) Juegos introductorios de presentación.

b) Introducción a las "ideas poderosas" de la ingeniería, robótica y programación a través de retos.

c) Trabajo individual o grupal.

d) Presentación y compartición con el grupo de la actividad realizada.

e) Exploración y juego libre.

Las sesiones fueron grupales dentro del horario habitual del aula hospitalaria ( 9 a 14h) y dentro su programación oficial de actividades, ya que el horario para actividades extracurriculares se realiza a partir de las $15 \mathrm{~h}$. Las actividades fueron llevadas a cabo por las investigadoras y la maestra responsable del aula. Dado que los participantes podían repetir las sesiones, se diseñaron diferentes actividades para trabajar dentro de una sesión, que trabajaran las competencias emocionales y de pensamiento computacional con el kit de robótica educativo KIBO.

Las actividades se relacionaron con las emociones y buscaron desarrollar la creatividad de los menores, así como el conocimiento sobre la cultura local. En la Tabla 1 se presentan algunos ejemplos de las actividades llevadas a cabo en el aula hospitalaria con KIBO en donde relacionamos la enseñanza del pensamiento computacional con las emociones. 
CARINA S. GONZÁLEZ-GONZÁLEZ, VERÓNICA VIOLANT HOLZ, ALFONSO INFANTE MORO,

Tabla 1

Ejemplos de diferentes actividades llevadas a cabo en las sesiones

\begin{tabular}{|c|c|c|c|}
\hline Nombre & Características & Descripción & Competencias \\
\hline Enfadator & $\begin{array}{l}\text { Objetivos: } \\
\text { 1. Aprender y apli- } \\
\text { car el proceso } \\
\text { de ingeniería } \\
\text { 2. Aprender los di- } \\
\text { ferentes com- } \\
\text { ponentes de un } \\
\text { robot y cómo } \\
\text { funcionan. } \\
\text { 3. Adquirir las } \\
\text { competencias } \\
\text { de pensamiento } \\
\text { computacional } \\
\text { Contenidos: } \\
\text { - Conocimiento } \\
\text { de ingeniería y } \\
\text { robótica } \\
\text { - Conocimiento } \\
\text { emocional } \\
\text { Duración: 30” } \\
\text { Materiales: } \\
\text { - Kit de robótica } \\
\text { KIBO } \\
\text { - Cuento “Enfa- } \\
\text { dator" } \\
\text { Materiales para } \\
\text { dibujar y pintar }\end{array}$ & $\begin{array}{l}\text { Se lee el cuento de "Enfada- } \\
\text { tor". Finalizada la lectura, se } \\
\text { les pregunta sobre qué creen } \\
\text { ellos que es un robot y para } \\
\text { qué creen que se utilizan los } \\
\text { robots según su propia opi- } \\
\text { nión. Después, se presenta a } \\
\text { KIBO y sus principales com- } \\
\text { ponentes como son el es- } \\
\text { queleto, los sensores, los co- } \\
\text { nectores, los mecanismos } \\
\text { móviles, la unidad de control } \\
\text { y la fuente de alimentación. } \\
\text { Esta explicación de los com- } \\
\text { ponentes de KIBO se lleva a } \\
\text { cabo comparando los com- } \\
\text { ponentes con las partes del } \\
\text { cuerpo humano para que los } \\
\text { participantes puedan relacio- } \\
\text { narlos. Cuando conozcan to- } \\
\text { das las partes de KIBO, se les } \\
\text { pide que dibujen su propio } \\
\text { robot en un folio en blanco } \\
\text { con bolitas de papel de seda } \\
\text { de diferentes colores. Cuando } \\
\text { todos tengan acabados sus } \\
\text { robots se los enseñarán al } \\
\text { resto de sus compañeros para } \\
\text { que puedan verlos. }\end{array}$ & $\begin{array}{l}\text { PC1 } \\
\text { PC4 }\end{array}$ \\
\hline
\end{tabular}




\begin{tabular}{|c|c|c|c|}
\hline Nombre & Características & Descripción & Competencias \\
\hline $\begin{array}{l}\text { Un circuito } \\
\text { emocional } \\
\text { para KIBO }\end{array}$ & $\begin{array}{l}\text { Objetivos: } \\
\text { 2. Aprender los di- } \\
\text { ferentes com- } \\
\text { ponentes de un } \\
\text { robot y cómo } \\
\text { funcionan. } \\
\text { 3. Aprender las } \\
\text { instrucciones de } \\
\text { programación. } \\
\text { 4. Adquirir las } \\
\text { competencias } \\
\text { de pensamiento } \\
\text { computacional } \\
\text { Contenidos: } \\
\text { - Conocimiento } \\
\text { de ingeniería y } \\
\text { robótica } \\
\text { - Conocimiento } \\
\text { del lenguaje de } \\
\text { programación } \\
\text { de KIBO } \\
\text { - Conocimiento } \\
\text { emocional } \\
\text { Duración: } 30 \text { ' } \\
\text { Materiales: } \\
\text { - Kit de robótica } \\
\text { KIBO } \\
\text { Imágenes de las } \\
\text { emociones de la } \\
\text { película Inside } \\
\text { Out }\end{array}$ & $\begin{array}{l}\text { Se presentará a KIBO y sus } \\
\text { principales componentes } \\
\text { como son el cuerpo, los sen- } \\
\text { sores, los conectores, los me- } \\
\text { canismos móviles, la unidad } \\
\text { de control y la fuente de ali- } \\
\text { mentación. Esta explicación } \\
\text { de los componentes de KIBO } \\
\text { se lleva a cabo comparando } \\
\text { sus componentes con las par- } \\
\text { tes del cuerpo humano para } \\
\text { que los participantes puedan } \\
\text { relacionarlos. Cuando conoz- } \\
\text { can todas las partes de KIBO, } \\
\text { se pasa a explicarles cómo } \\
\text { pueden programar sus dife- } \\
\text { rentes movimientos mediante } \\
\text { el lector de códigos de barras } \\
\text { de los cubos. Dependiendo de } \\
\text { la edad de los niños, niñas y } \\
\text { jóvenes presentes, se expli- } \\
\text { can las programaciones más } \\
\text { básicas o las más complejas. } \\
\text { Una vez que sepan cómo pro- } \\
\text { gramar a KIBO se les deja } \\
\text { que prueben para familiari- } \\
\text { zarse con él y su programa- } \\
\text { ción. Cuando todos conoz- } \\
\text { can a KIBO y su manejo, se } \\
\text { les dan las imágenes de las } \\
\text { emociones del vídeo traba- } \\
\text { jado anteriormente para que } \\
\text { las coloquen por diferentes } \\
\text { lugares del suelo del aula. A } \\
\text { continuación, se les pedirá } \\
\text { que programen a KIBO para } \\
\text { que llegue a cada una de las } \\
\text { emociones que se les pida. } \\
\text { Este proceso se puede repe- } \\
\text { tir varias veces para que to- } \\
\text { dos puedan participar progra- } \\
\text { mando a KIBO. }\end{array}$ & $\begin{array}{l}\text { PC1 } \\
\text { PC2 } \\
\text { PC3 } \\
\text { PC4 } \\
\text { PC5 } \\
\text { PC6 }\end{array}$ \\
\hline
\end{tabular}


CARINA S. GONZÁLEZ-GONZÁLEZ, VERÓNICA VIOLANT HOLZ, ALFONSO INFANTE MORO,

\begin{tabular}{|c|c|c|c|}
\hline Nombre & Características & Descripción & Competencias \\
\hline Paseando & $\begin{array}{l}\text { Objetivos: } \\
\text { 2. Aprender los di- } \\
\text { ferentes com- } \\
\text { ponentes de un } \\
\text { robot y cómo } \\
\text { funcionan. } \\
\text { 3. Aprender las } \\
\text { instrucciones de } \\
\text { programación. } \\
\text { 4. Adquirir las } \\
\text { competencias } \\
\text { de pensamiento } \\
\text { computacional } \\
\text { Contenidos: } \\
\text { - Conocimiento } \\
\text { del lenguaje de } \\
\text { programación } \\
\text { de KIBO } \\
\text { - Conocimiento } \\
\text { emocional } \\
\text { Duración: } 30 \text { ' } \\
\text { Materiales: } \\
\text { - Kit de robótica } \\
\text { KIBO } \\
\text { Música }\end{array}$ & $\begin{array}{l}\text { Se explica cómo pueden pro- } \\
\text { gramar diferentes movimien- } \\
\text { tos con KIBO. Dependiendo } \\
\text { de la edad de los niños, niñas } \\
\text { y jóvenes presentes, se expli- } \\
\text { can las programaciones más } \\
\text { básicas o las más complejas. } \\
\text { Una vez que sepan cómo pro- } \\
\text { gramar a KIBO se les deja } \\
\text { que prueben para familiari- } \\
\text { zarse con él y su programa- } \\
\text { ción. Cuando todos conoz- } \\
\text { can como programar a KIBO } \\
\text { se habilita un lugar para que } \\
\text { pueda pasear. Se les pondrá } \\
\text { música y se les irá pidiendo } \\
\text { que mientras pasean se mue- } \\
\text { van según las instrucciones } \\
\text { que se les digan. Algunas de } \\
\text { estas instrucciones pueden } \\
\text { ser: alegres, enfadados, tris- } \\
\text { tes, asqueados, miedosos, } \\
\text { cantando, pesados, modelos, } \\
\text { despacio, a cámara lenta, ro- } \\
\text { bot, animales, como sugie- } \\
\text { ran los compañeros, imitando } \\
\text { a KIBO, etc. Mientras y por } \\
\text { turnos irán programando a } \\
\text { KIBO para que pase con ellos } \\
\text { llevando a cabo las instruc- } \\
\text { ciones antes mencionadas. }\end{array}$ & $\begin{array}{l}\text { PC2 } \\
\text { PC3 } \\
\text { PC4 } \\
\text { PC5 } \\
\text { PC6 }\end{array}$ \\
\hline
\end{tabular}

Competencias de Pensamiento Computacional (PC):

PC1: hardware/software (los objetos inteligentes, no son mágicos, sino que son creados por la ingeniería humana)

PC2: algoritmos (secuenciación, orden, organización lógica)

PC3: modularidad (descomposición de tareas, instrucciones), estructuras de control (reconocimiento de patrones, repetición, causa y efecto)

PC4: representación (representación simbólica, modelos)

PC5: proceso de diseño (resolución de problemas, iteración y perseverancia, edición/revisión)

PC6: depuración (identificación de problemas, resolución de problemas, iteración y perseverancia) 


\section{Instrumentos de recogida de datos}

Para la evaluación de cada una de las sesiones se utilizaron los siguientes instrumentos:

- Emodiana (González-González et al., 2013). La Emodiana (Figura 1) permite identificar una o más emociones, ya que los participantes pueden sentirse por ejemplo con miedo, pero al mismo tiempo ansiosos, o sentir alegría y al mismo tiempo sorpresa o satisfacción. Además, permite registrar y categorizar las razones de las emociones en función de si son inherentes a la persona, debidas a la estructura de la actividad o producidas por razones externas. Las emociones registradas con esta herramienta pueden ser categorizadas en positivas, negativas y neutras, de acuerdo con trabajos previos (González-González et al., 2017). Es con estas categorías con las que revisaremos el impacto emocional de la intervención realizada. A través de este instrumento podemos identificar las emociones de los participantes antes y después de la sesión. De esta forma, una investigadora recoge las emociones antes y después de la intervención con información cuantitativa (qué emoción es identificada e intensidad de la emoción percibida por el sujeto) e información cualitativa (explicación de por qué se percibe dicha emoción). La información cualitativa es categorizada en tres categorías de posibles razones causales de la emoción: persona, actividad y contexto. Aunque la calidad de la información cualitativa varía según la edad del sujeto respondiente y su inteligencia emocional, la Emodiana favorece la identificación de las emociones a través de las representaciones gráficas. Esto hace que sea un instrumento que pueda ser aplicado en edades infantiles y en la adolescencia. Asimismo, el instrumento permite identificar más de una emoción.

- Kit de robótica KIBO. Es un robot educativo tangible creado especialmente para la enseñanza de la programación y pensamiento computacional, por el Grupo de Investigación de Tecnologías del Desarrollo en la Universidad de Tufts a través de fondos de la Fundación Nacional de Ciencia (NSF) (Sullivan et al., 2015). KIBO es un kit de construcción de robótica de 18 bloques que incluye hardware (el propio robot) y software (bloques de programación tangibles) que se utilizan para hacer que el robot se mueva. Los bloques de programación son de madera y no contienen componentes electrónicos ni componentes digitales integrados. El kit contiene diferentes elementos con los cuales se puede construir el robot fácilmente, tales como: ruedas, motores, salida de luz y una 
CARINA S. GONZÁLEZ-GONZÁLEZ, VERÓNICA VIOLANT HOLZ, ALFONSO INFANTE MORO,

variedad de sensores que permite que los niños y niñas aprendan diferentes conceptos de programación, desde sencillos a complejos, incluyendo bucles de repetición, declaraciones condicionales y declaraciones de anidamiento (Figuras 2 y 3) (Bers et al., 2019).

- Rúbrica de evaluación de las competencias relacionadas con el pensamiento computacional y un diario de las sesiones. En base a las habilidades propuestas por Bers (2018), se elaboró una rúbrica para verificar si los participantes de las sesiones habían conseguido los objetivos relacionados con el aprendizaje del pensamiento computacional. Esta rúbrica fue completada por los investigadores participantes del estudio, seguiendo una plantilla (Tabla 2).

- Diario de las sesiones. Se registraron los momentos importantes y las reflexiones personales de los investigadores sobre las actividades de robótica de KIBO que se llevan a cabo en cada una de las sesiones. La información que se recoge es la siguiente: fecha de la sesión, nombre y apellidos del docente hospitalario, nombre y apellidos de las personas que desarrollaron la intervención, descripción de los objetivos conseguidos, los momentos difíciles y momentos significativos de la sesión, las herramientas y estrategias pedagógicas implementadas y las adaptaciones que se realizaron en la sesión.

Figura 1

Instrumento "Emodiana"

(González et al., 2013)

\section{MIMDIANA}

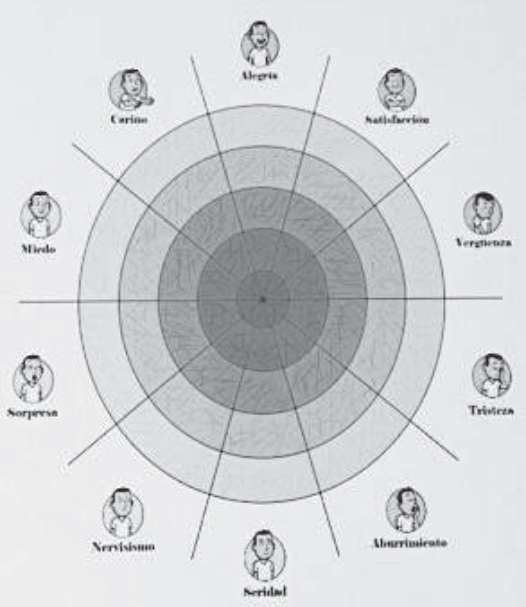


Figura 2

Kit del Robot KIBO utilizado en el estudio

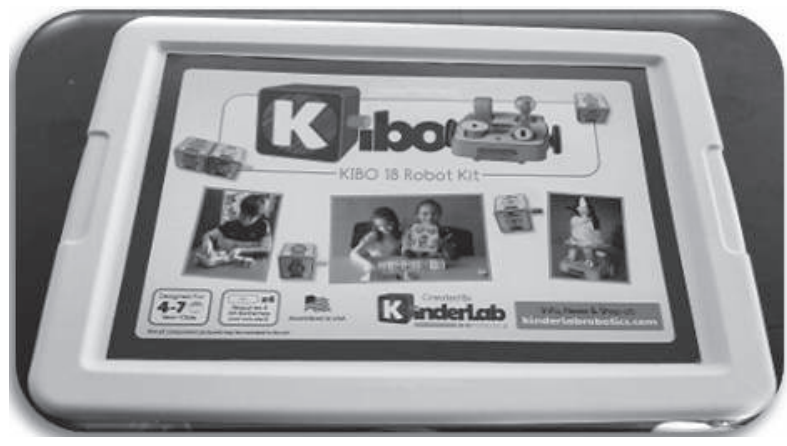

Fuente: fotografía propia del kit

Figura 3

Programación y lectura realizada a través del escáner de KIBO

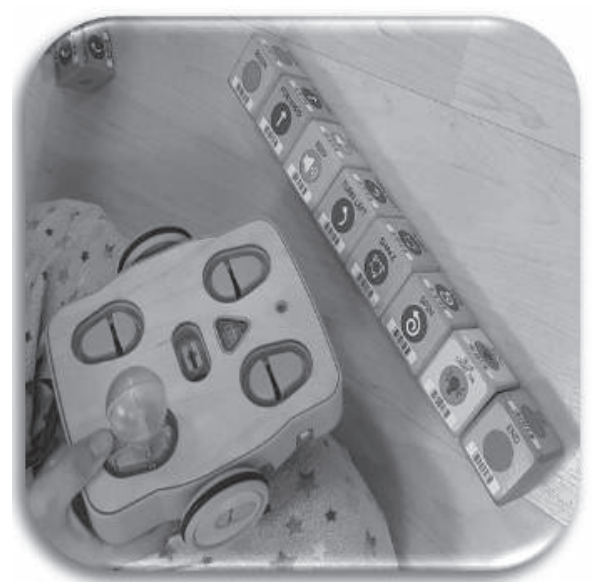

Fuente: fotografía propia

Tabla 2

Rúbrica para evaluación de la sesión

\section{Competencias relacionadas con el Pensamiento Computacional}

$\begin{array}{lllll}1 & 2 & 3 & 4 & 5\end{array}$

PC1: Comprende el hardware y software de KIBO

PC2: Realiza algoritmos con los bloques de KIBO (secuenciación, orden, organización lógica) 


\section{Competencias relacionadas con el Pensamiento Computacional}

$\begin{array}{lllll}1 & 2 & 3 & 4 & 5\end{array}$

PC3: Crea programas con el lenguaje de KIBO modularmente y con estructuras de control

PC4: Identifica los diferentes bloques de programación de

KIBO (representación simbólica, modelos)

PC5: Aplica correctamente el proceso de diseño en sus tareas (resolución de problemas, iteración y perseverancia, edición/ revisión)

PC6: Depura sus soluciones (identificación de problemas, resolución de problemas, iteración y perseverancia)

$1=$ Totalmente en desacuerdo/ $5=$ Totalmente de acuerdo

\section{ANÁLISIS Y RESULTADOS}

\section{Emodiana: estados emocionales relacionados con la intervención}

El análisis de las emociones referidas por los/as participantes a la entrada y a la salida de las sesiones grupales nos interesa especialmente por el carácter informativo sobre el impacto emocional de la misma. Así, para valorar el efecto emocional de la introducción de KIBO en el aula hospitalaria, compararemos las emociones informadas antes de iniciar las sesiones con las de la finalización de estas. Trabajamos con el total de valoraciones tomadas a lo largo de las cinco sesiones realizadas.

La Figura 4 presenta los resultados obtenidos sobre las emociones percibidas por los niños y niñas al llegar al aula hospitalaria, antes de comenzar las sesiones. En la Figura 8, se exponen los resultados sobre las emociones manifestadas por los niños y niñas al finalizar las sesiones, al irse del aula hospitalaria después de la sesión de intervención con KIBO.

Como puede observarse, las emociones que más frecuentemente se dieron antes de las sesiones eran: miedo (24\%), alegría (21\%), tristeza $(16 \%)$, nerviosismo $(16 \%)$, vergüenza $(16 \%)$, cariño $(3 \%)$, aburrimiento $(2 \%)$ y seriedad (2\%). Podemos ver que en un 58\% eran emociones negativas (tristeza, miedo, aburrimiento, nerviosismo), un 24\% de emociones positivas (alegría y cariño) y un 18\% neutras (vergüenza y seriedad). 
Figura 4

Resultados totales sobre las emociones percibidas antes de la sesión

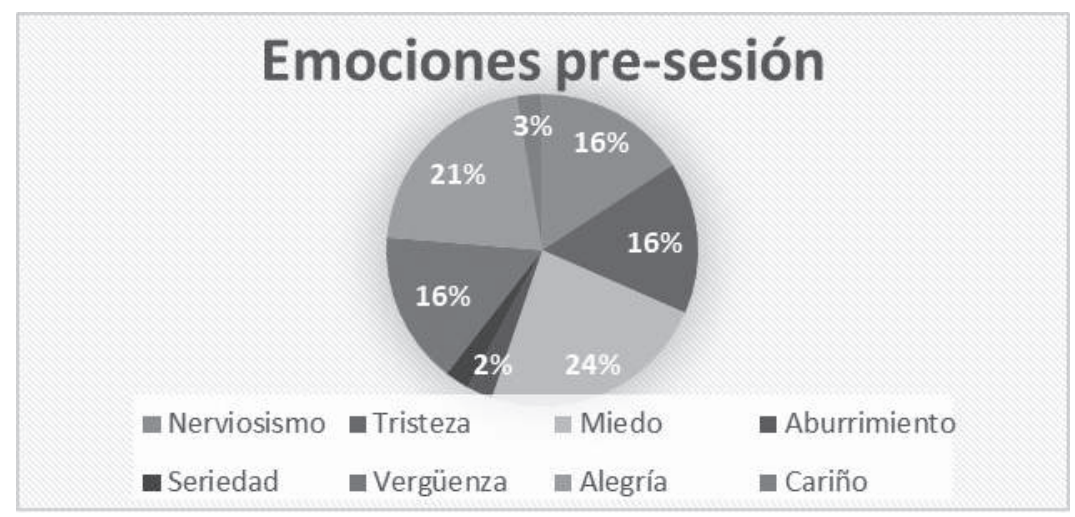

También se les preguntó a los menores sobre las razones de por qué se sentían de esa manera, no solo por el tipo de emoción de la Emodiana. Por ejemplo, en el caso de la alegría, los niños y niñas dijeron que estaban felices por salir de sus habitaciones o por poder reunirse con otros niños y niñas en el aula. En cambio, en el caso del aburrimiento era debido a la falta de entretenimiento en sus habitaciones, miedo y nerviosismo por la operación o tristeza por no poder salir del hospital (Figura 5).

\section{Figura 5}

Imágenes de la sesión sobre emociones en dónde se muestran dibujos realizados por los niños y niñas expresando sus emociones y posterior programación hacia las emociones identificadas
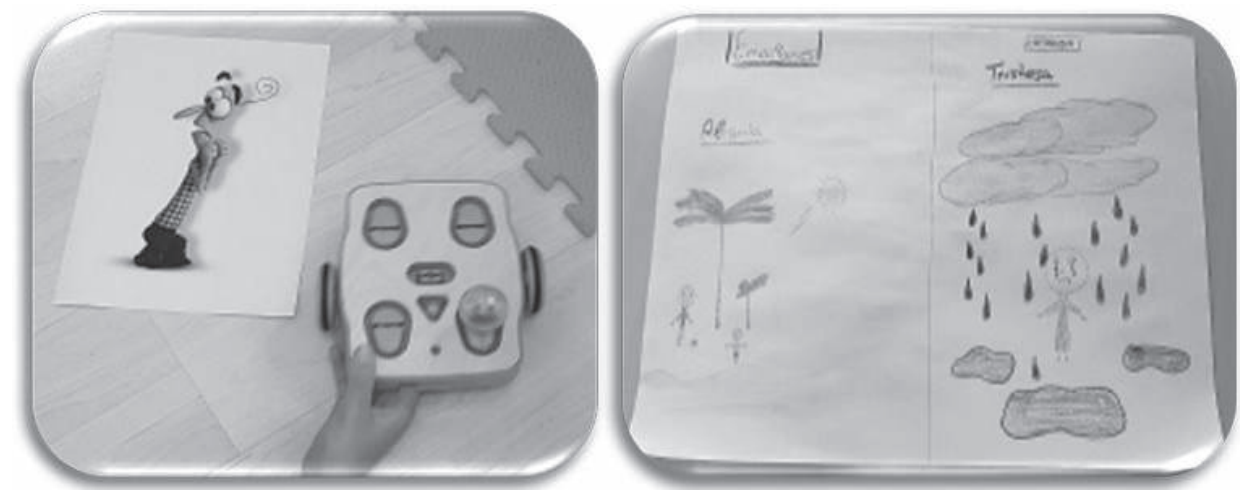

En la Figura 6, se exponen los resultados sobre las emociones manifestadas por los niños y niñas al finalizar las sesiones, al irse del aula hospitalaria después de la sesión de intervención con KIBO. Podemos observar (figura 6) que las emociones que expresaron los menores después 
de las sesiones fueron: alegría (43\%), sorpresa (11\%), satisfacción (11\%), cariño $(11 \%)$, nerviosismo (7\%), tristeza (7\%), miedo (7\%) y seriedad (3\%). Vemos cómo aumentan las emociones positivas (65\%) frente a las emociones negativas $(21 \%)$ y neutras (14\%). Además, vemos que aparecen al finalizar la sesión nuevas emociones como la sorpresa y la satisfacción. Como razones respecto a estas dos nuevas emociones los niños y niñas expresaron que no esperaban haberse divertido o sorprendido con las actividades realizadas durante la sesión. Además, aumentó la alegría que, en este caso, tal y como lo manifestaron los niños y niñas, estaba relacionado con la propia actividad realizada en la sesión. Asimismo, disminuyeron el nerviosismo, la tristeza y el miedo y desaparecieron emociones como el aburrimiento y la vergüenza.

Figura 6

Resultados totales sobre las emociones percibidas después de la sesión

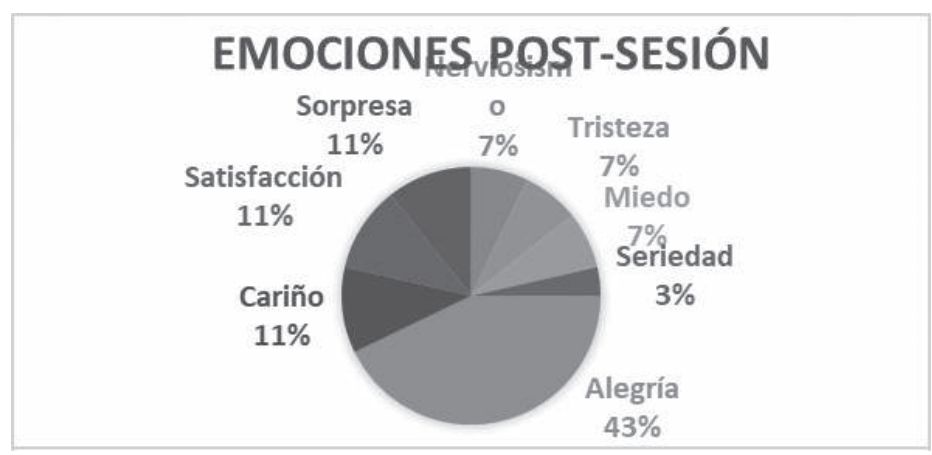

En la Figura 7 podemos observar las emociones categorizadas en positivas, negativas y neutras de forma comparada de los momentos antes y después de la sesión.

Figura 7

Número de emociones clasificadas en cada categoría al finalizar las sesiones en función de la clasificación de a la entrada

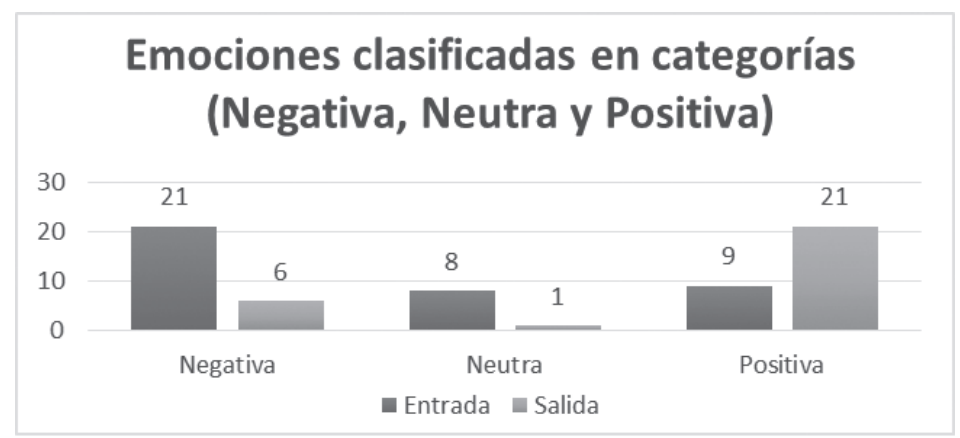


La Tabla 3 presenta la tabla de contingencias que recoge el porcentaje de casos que informan cada emoción de salida en función de la emoción de entrada. En dicha tabla observamos que el 100\% de los casos en que se informó emociones positivas al inicio, refieren emociones positivas a la salida; el $71.43 \%$ de los casos de emociones negativas a la entrada se modifican a positivas a la salida, el $23.80 \%$ conservaron su estado negativo y un $4.77 \%$ pasaron a emociones neutras; por otra parte, el $90 \%$ de los casos que informaron emociones neutras al inicio lo mantuvieron y pasaron a emociones negativas en un $10 \%$ de los casos.

Tabla 3

Tabla de contingencia para las emociones

\begin{tabular}{ccccc}
\hline & & \multicolumn{3}{c}{ Emoción Salida } \\
& & Positiva & Neutra & Negativa \\
\hline & Neutra & $0 \%$ & $90 \%$ & $10 \%$ \\
Emoción & Positiva & $100 \%$ & $0 \%$ & $0 \%$ \\
Entrada & Negativa & $71.43 \%$ & $4.77 \%$ & $23.80 \%$ \\
\hline
\end{tabular}

El análisis estadístico confirma que las emociones indicadas por los menores antes de la intervención y después de la intervención son estadísticamente significativas, como se demuestra a través de la prueba chi-cuadrado $\left(\chi^{2}(2)=.001 ; \mathrm{p} \leq .001\right)$.

\section{Evaluación del pensamiento computacional}

Las seis competencias relacionadas con el pensamiento computacional fueron puntuadas por los investigadores utilizando una escala del 1-5, donde el valor más alto implica un mayor grado de dominio observado en la competencia relacionada. Se realizó una rúbrica por participante y sesión y luego se obtuvo la media de las puntuaciones obtenidas.

Los resultados muestran que las competencias relacionadas con el pensamiento computacional que mejor han sido logradas fueron la PC1 (hardware y software) y la PC2 (algoritmos) $(M=4.2)$, seguidas de la PC4 (representación) $(M=4)$, la PC3 (modularidad) $(M=3.7)$ y la PC5 (proceso de diseño) $(M=3.4)$. La competencia que parece resultar más difícil de lograr es la PC6 (depuración) $(M=2.9)$ (Ver Figura 8). 
CARINA S. GONZÁLEZ-GONZÁLEZ, VERÓNICA VIOLANT HOLZ, ALFONSO INFANTE MORO,

Figura 8

Puntuación media obtenida de la rúbrica de evaluación sobre pensamiento computacional

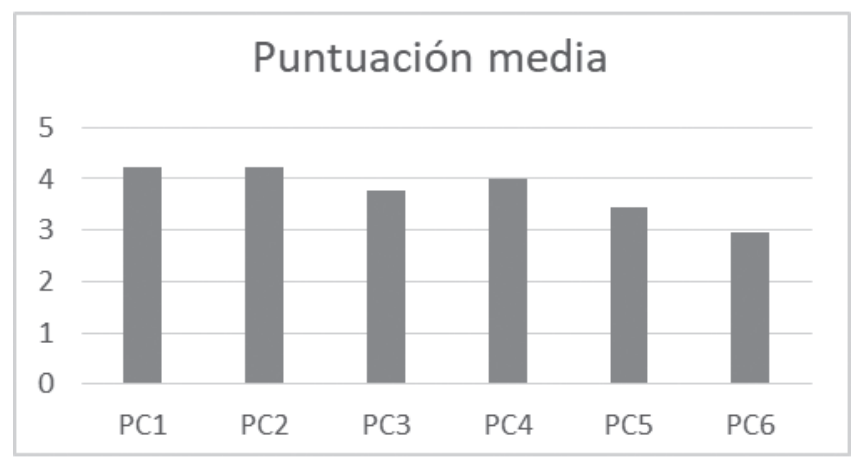

\section{Diario de sesiones}

La información recogida en el diario de sesiones recoge la efectividad de la intervención realizada con el alumnado hospitalizado. Los participantes se han mostrado muy dispuestos y participativos en todas las actividades. Los registros cualitativos de las sesiones indican un alto grado de cumplimiento de los objetivos planteados en las actividades de robótica educativa planteadas.

Sin embargo, la planificación inicial de las sesiones tuvo que ser adaptada en cada caso, dado que una de las características principales de las aulas hospitalarias es que no es posible conocer quiénes vendrán a la sesión y qué necesidades particulares tendrán hasta el momento de esta. Por tanto, se tuvieron que realizar las adaptaciones necesarias en función de las necesidades y características de cada persona relacionadas a su situación particular, además de las diferentes edades de los participantes asistentes y a que podían entrar o salir de la sesión en cualquier momento debido a sus tratamientos. Por ejemplo, en la sesión 1, uno de los participantes iba en silla de ruedas, por lo que todos los participantes se sentaron en sillas formando un círculo para las actividades de presentación. También, las actividades tuvieron que adaptarse a la llegada de un nuevo participante en el medio de la sesión, para que pudiera participar con el resto de sus compañeros.

Otras adaptaciones fue el ofrecer ayuda a los participantes cuando tenían vías o estaban con suero, ya que no podían manipular con facilidad los bloques y escanear los códigos con el robot. En estos casos, uno de los investigadores participantes ofrecía ayuda personalizada para construir el robot, armar las secuencias en función de las instrucciones del niño/a y escanear la misma. 
Por otra parte, el trabajo con las emociones provocó muchas situaciones significativas durante las sesiones, ya que afloraban durante la actividad y podían hablar con otros niños y niñas, además de con las investigadoras y la docente hospitalaria sobre cómo se sentían. Todo ello generó un clima de complicidad y de apoyo mutuo.

\section{CONCLUSIONES}

Las aulas hospitalarias están concebidas con el fin de continuar el desarrollo educativo, afectivo y social de los niños, niñas y jóvenes que se encuentran hospitalizados, siempre y cuando su estado médico lo permita. Con respecto a las sesiones llevadas a cabo, podemos afirmar que se han conseguido los objetivos establecidos para cada una de las mismas. Las estrategias pedagógicas que se han utilizado en las sesiones son el trabajo individual, el trabajo cooperativo, el aprendizaje por descubrimiento, el juego y el trabajo creativo.

En relación con la pregunta de investigación sobre si es posible introducir la enseñanza del pensamiento computacional de forma transversal a través de la robótica educativa en las aulas hospitalarias, la respuesta es positiva. Vemos que las seis competencias sobre pensamiento computacional evaluadas a través de la rúbrica alcanzaron puntuaciones medias satisfactorias. El alumnado es capaz de comprender el hardware y software del robot, pueden pensar y ejecutar algoritmos con los bloques de programación, identificar y diferenciar las diferentes instrucciones de los bloques y programar modularmente, realizando tareas correctivas.

Las puntuaciones obtenidas pueden justificarse por la facilidad que supone trabajar con un kit de robótica creado para edades comprendidas entre 4 y 7 años, cuando la media de edad del estudio fue de 12.4. Sin embargo, la elección del kit se justifica debido a que la variabilidad de edades del aula hospitalaria oscila entre 0 y 16 años, y se deseaba crear una propuesta inclusiva en donde niños y niñas, y jóvenes de diferentes edades pudieran participar. En este sentido, KIBO cumplió las expectativas con creces. Además, deseamos no aumentar el tiempo de exposición a las pantallas de los menores, y con este kit de robótica educativa, que no requiere ordenador ni tabletas, se puede trabajar el pensamiento computacional de forma tangible y desconectada.

Sobre la pregunta de si existen diferencias significativas entre las emociones de entrada y salida después de la intervención con KIBO en el aula hospitalaria, a través de los resultados obtenidos del registro de la Emodiana podemos demostrar que existen diferencias significativas entre 
CARINA S. GONZÁLEZ-GONZÁLEZ, VERÓNICA VIOLANT HOLZ, ALFONSO INFANTE MORO,

las emociones indicadas por los menores a la entrada y a la salida del aula hospitalaria. Además, observamos que la introducción de KIBO en el aula hospitalaria aumenta las emociones positivas frente a las negativas o neutras. Aunque algunas emociones permanezcan al inicio y final de la sesión, se observan diferencias en las intensidades. Por ejemplo, en una de las sesiones tres participantes aumentan la intensidad de alegría que tenían al principio de la sesión por las actividades llevadas a cabo, y otro de los participantes ha añadido el cariño al final de la sesión por hacer nuevos amigos y amigas durante la sesión. Y otro de los participantes disminuye la intensidad de nerviosismo que presentaba al principio de la sesión por la fecha próxima a la operación que tenía programada. Además, aparecen otras emociones positivas no presentes al inicio de la sesión, como la alegría, la satisfacción y el cariño por las actividades llevadas a cabo durante la sesión.

Muchos de los participantes no sabían muy bien explicar por qué sentían esas emociones y con esa intensidad y algunos de ellos no tenían la confianza suficiente como para explicar las razones de por qué sentían lo que sentían, y simplemente nos decían que no sabían o que no podían decírnoslo. En algunos casos, algunos participantes tuvieron que irse antes de finalizar la sesión, y por eso no se pudo recoger qué emociones sentían al finalizarla.

Por todo lo expuesto, la propuesta de intervención parece favorecer emociones positivas, pues cuando al análisis anterior unimos el de sus justificaciones, vemos que estas tienden a relacionarse con los elementos de la actividad (juegos, participantes, compañeras, aprendizajes), además de hacer referencia a motivos personales. Entre estos últimos destacan verbalizaciones relacionadas con divertirse, pasarlo bien y salir de la habitación para hacer nuevos amigos/as. También, al aparecer la emoción de satisfacción al final de la sesión, podemos concluir que la intervención favorece autopercepciones positivas.

Para finalizar, como limitaciones de este estudio debemos mencionar la variabilidad de la muestra participante y la imposibilidad de hacer un seguimiento a largo plazo sobre los aprendizajes del alumnado del aula hospitalaria, debido a su propio contexto y situación, donde las estancias en el hospital tienen a ser cada vez menores y sus propias patologías y tratamientos son muy particularizados. Como trabajos futuros, se pretende continuar la investigación sobre el impacto de la robótica en las emociones de los menores hospitalizados y el fomento de vocaciones STEAM en el aula hospitalaria. 


\section{REFERENCIAS BIBLIOGRÁFICAS}

Antequera, J.G. y Domínguez, F.I.R. (2015). Videojuegos precursores de emociones positivas: propuesta metodológica con Minecraft en el aula hospitalaria. IJERI: International Journal of Educational Research and Innovation, 3, 105-120.

Area, M. (2015). La escuela en la encrucijada de la sociedad digital. Cuadernos de pedagogía, 462, 26-31.

Artioli, C.L. (2018). Educação hospitalar: uma questão de direito. Revista actualidades investigativas en educación, 19(1), 1-18. https://doi. org/10.15517/aie.v19i1.35600

Asensio-Ramón, P. (2018). La Pedagogía Hospitalaria y los Derechos del Niño: un camino recorrido y por recorrer. XVI Congreso Nacional Educación Comparada, Tenerife, 25-30. http://doi. org/10.25145/c.educomp.2018.16.003

Balanskat, A. \& Engelhardt, K. (2015). Computing our future: Computer programming and coding. Priorities, school curricula and initiatives across Europe. Technical Report, European Schoolnet. shorturl.at/bjryE

Barquero, A. (2017). La inteligencia emocional a través de la robótica en las aulas hospitalarias. EDHOSPI. Revista divulgativa sobre educación hospitalaria, 5, 41-44.

Bers, M.U. (2018). Coding as a Playground: Programming and Computational Thinking in the Early Childhood Classroom. Routledge Press.

Bers, M.U. (2008). Blocks, robots and computers: Learning about technology in early childhood. Teacher's College Press.

Bers, M.U., González-González, C., \& Armas-Torres, M. B. (2019). Coding as a playground: Promoting positive learning experiences in childhood classrooms. Computers \& Education, 138, 130-145. https://doi.org/10.1016/j. compedu.2019.04.013

Bisquerra, R. (2015). Competencia emocional. En M. Alguacil (Ed.), Perfil competencia del profesional que interviene durante la enfermedad y convalecencia (pp. 47-61). Ediciones Aljibe.

Bocconi, S., Chioccariello, A., Dettori, G., Ferrari, A., \& Engelhardt, K. (2016). Developing computational thinking in compulsory education - Implications for policy and Practice. Publications Office of the European Union. http:// dx.doi.org/10.2791/792158

Calvo, M.I. (2017). La pedagogía hospitalaria: clave en la atención al niño enfermo y hospitalizado y su derecho a la educación. Aula 23, 33-47. http://dx.doi.org/10.14201/ aula2017233347

Castellanos, S.A.P., y Torres, L.S.Q. (2015). Propuesta para el apoyo pedagógico y del manejo de las emociones a niños hospitalizados por periodos de tiempo largo o recurrente. Congreso Internacional Infancia en Contextos de Riesgo, 2401-2405

Dawe, J., Sutherland, C., Barco, A., \& Broadbent, E. (2019). Can social robots help children in healthcare contexts? A scoping review. BMJ paediatrics open, 3(1), 1-16. https://doi. org/10.1136/bmjpo-2018-000371

De Mula-Fuentes, B., Quintana, M., Rimbau, J., Martínez-Mejías, A., Socorro, M., Rivera-Pérez, C., y Garolera, M. (2018). Ansiedad, miedos hospitalarios y alteraciones conductuales en la hospitalización 
CARINA S. GONZÁLEZ-GONZÁLEZ, VERÓNICA VIOLANT HOLZ, ALFONSO INFANTE MORO, LORENA CÁCERES GARCÍA, MARÍA DOLORES GUZMÁN FRANCO

ROBÓTICA EDUCATIVA EN CONTEXTOS INCLUSIVOS: EL CASO DE LAS AULAS HOSPITALARIAS

infantil. Actas Españolas de Psiquiatría, 46(2), 42-50.

Dirección General de Ordenación, Innovación y Promoción Educativa. Consejería de Educación y Universidades (2018). BOC $N^{\circ} 108$. 2658. Resolución de 22 de mayo de 2018, por la que se dictan instrucciones para la organización de la respuesta educativa al alumnado de la Educación Infantil, la Enseñanza Básica y el Bachillerato [...] en la Comunidad Autónoma de Canarias. https://bit. ly/3hjnw74

Dufva, T., \& Dufva, M. (2019). Grasping the future of the digital society. Futures, 107, 17-28. https://doi. org/10.1016/j.futures.2018.11.001

Ramos, J.R., Montiano, J.I., Hernández Marco, R., \& García García, J.J. (2014, November). Situación de la pediatría hospitalaria en España: informe de la Sociedad Española de Pediatría Hospitalaria (SEPHO). Anales de Pediatría, 81(5), 326-e1-326.e8. https:// doi.org/10.1016/j.anpedi.2014.01.006

García-Peñalvo, F.J. (2017). Pensamiento computacional en los estudios preuniversitarios. El enfoque de TACCLE3. https://doi.org/10.5281/ zenodo.376310-

González-González, C.S., CáceresGarcía, L., \& Violant-Holz, V. (2019). Bringing Computational Thinking to Hospital Classrooms. In Proceedings of the Seventh International Conference on Technological Ecosystems for Enhancing Multiculturality (pp. 31-35). ACM. https://doi. org/10.1145/3362789.3362908

Gonzalez-González, C.S., Toledo, P., Alayon, S., Munoz, V., \& Meneses D. (2011). Using Information and Communication Technologies in Hospital Classrooms: SAVEH
Project. Knowledge Management \& E-Learning: An International Journal, 3(1), 72-83. https://doi.org/10.34105/j. kmel.2011.03.008

González-González, C.S. (2015). Competencia digital. En V. Violant (Coord.) y M. Alguacil (Ed.), Perfil competencia del profesional que interviene durante la enfermedad y convalecencia (pp. 183-206). Ediciones Aljibe.

González-González, C.S., y Violant, V. (Ed.) (2015). Uso de las TIC para la atención educativa, hospitalaria y domiciliaria. Mc Graw Hill.

González-González, C.S., NavarroAdelantado, V., y Cairós-González, M. (2017). Fiabilidad de un instrumento de evaluación emocional a través de la concordancia inter-observador para el aprendizaje basado en juegos. Actas del V Congreso Internacional de Videojuegos y Educación (CIVE'17). Puerto de la Cruz, Tenerife. https://bit. $\mathrm{ly} / 39 \mathrm{csPm} 4$

González-González, C.S., Ramírez, R.V., \& Holz, V.V. (2019, October). A MOOC for crossing boundaries in hospital classrooms through ICT. 2019 IEEE Learning with MOOCS (LWMOOCS) (pp. 145149). IEEE. https://doi.org/10.1109/ LWMOOCS47620.2019.8939604

González-González, C.S., CairósGonzález, M., y Navarro-Adelantado, V. (2013). EMODIANA: Un instrumento para la evaluación subjetiva de emociones en niños y niñas. XIV Congreso Internacional de Interacción Persona-Ordenador (Vol. 2, No.5112.2169). https://doi. org/10.13140/RG.2.1.5112.2169

Guillén; M., y Mejia, A. (2002). Actuaciones educativas en Aulas 
CARINA S. GONZÁLEZ-GONZÁLEZ, VERÓNICA VIOLANT HOLZ, ALFONSO INFANTE MORO, LORENA CÁCERES GARCÍA, MARÍA DOLORES GUZMÁN FRANCO ROBÓTICA EDUCATIVA EN CONTEXTOS INCLUSIVOS: EL CASO DE LAS AULAS HOSPITALARIAS

Hospitalarias. Atención escolar a niños enfermos. Narcea.

Hen, M. (2020). Teaching Emotional Intelligence: An Academic Course for Hospital Teachers. Continuity in Education, 1(1), 22-36. https://doi. org/10.5334/cie.13

Hernández-Pérez, E. y Rabadán, J.A. (2014). "Érase una vez...un cuento curativo". Atención educativa en población infantil hospitalarizada a través de la literatura. Educatio Siglo XXI, 32(2), 129-150. https://doi. org/10.6018/j/194121

Lizasoàin, O. \& Polaino, A. (1995). Reduction of anxiety in pediatric patients: effect of a psychopedagogical intervention programme. Patient Educational and Coundeling, 25, 1722. https://doi.org/10.1016/07383991(94)00651-2

Lizasoáin, O., y Violant, V. (2015). Marco conceptual de la Pedagogía Hospitalaria y su aplicación desde una visión holística. En V. Violant (Ed.), Bases de la pedagogía hospitalaria aplicada a las etapas vitales (pp. 19-49). Ediciones Aljibe.

Llorens, F., García-Peñalvo F.J., Molero, X., y Vendrell, E. (2017). La enseñanza de la informática, la programación y el pensamiento computacional en los estudios preuniversitarios. Education in the Knowledge Society, 18(2), 7-17. https://doi.org/10.14201/ eks2017182717

Maher, D. (2020). Pre-Service Teachers' Digital Competencies to Support School Students' Digital Literacies. In Handbook of Research on Literacy and Digital Technology Integration in Teacher Education (pp. 29-46). IGI Global. https://doi.org/10.4018/978-17998-1461-0.ch002
Ministerio de Educación, Cultura y Deportes de España (2018). Programación, robótica y pensamiento computacional en el aula. Situación en España. https://bit.ly/3jokCQk

Morales, L.O. (2019). La pedagogía hospitalaria, un reto gigante para la virtualidad. Revista Reflexiones $y$ Saberes, 10, 17-24.

Naranjo, I.L. y Castillo, A.F. (2006). Hospitalización infantil y atención psico-educativa en contextos excepcionales de aprendizaje. Revista de educación, 341, 553-577.

Nuncira, T.A., Espitia, M.L.A., Mosos, J.S.L., Cubides, L.R.R., Ovalle, C.Y., Navarro, C.P.G., ... y de los Reyes, L.D.C. (2019). Implementación del método STEM a través de la robótica educativa como terapia coadyuvante para mejorar la calidad de vida en pacientes pediátricos oncológicos del Hospital Militar Central, Bogotá, Colombia. (Primera fase). Congreso Internacional de Tecnologías en la Educación. https://bit.ly/2WEFDwp

Ocampo, A. G., y Lizasoáin, O. R. (2019). Pedagogía hospitalaria: trayectorias de desarrollo intelectual, conquistas profesionales y desafíos de futuro. Revista Intersaberes, 13(29), 415-423.

Ortigosa JM, Quiles MJ, Méndez FX, y Pedroche S. Miedos infantiles hospitalarios. Un estudio con niños hospitalizados y no hospitalizados. Ansiedad y Estrés 2000, 6, 61-70.

Polaino-Lorente, A. y Lizasoàin, O. (1994). Programas de intervención y modificación del autoconcepto en niños hospitalizados. Revista de Psicología General y Aplicada, 47(3), 333-337.

Prendes, P. (2014). Ebook Pedagogía Hospitalaria y TIC. https://bit. ly/39cciyB 
CARINA S. GONZÁLEZ-GONZÁLEZ, VERÓNICA VIOLANT HOLZ, ALFONSO INFANTE MORO, LORENA CÁCERES GARCÍA, MARÍA DOLORES GUZMÁN FRANCO

ROBÓTICA EDUCATIVA EN CONTEXTOS INCLUSIVOS: EL CASO DE LAS AULAS HOSPITALARIAS

Pretz, K. (2014). Computer science classes for kids becoming mandatory. https://bit.ly/3eLyRLE

Resnick, M. (2013). Learn to Code, Code to Learn. EdSurge. https://bit. ly/2ZKcemv

Ryu, G.J., Kang, J.B., Kim, C.G., \& Song, B.S. (2013). Development of a robot remote support system for student with health impairment. Proceedings of the 7th International Convention on Rehabilitation Engineering and Assistive Technology (p.24). Singapore Therapeutic, Assistive \& Rehabilitative Technologies (START) Centre.

Romero, K. y Alonso, L. (2007). Un modelo de práctica pedagógica para las aulas hospitalarias: el caso del hospital universitarios de los Andes. Revista Central de Venezuela, 28(083), 407-441.

Sequeira, J.S., Ferreira, M.I.A., Barata, A.N., \& Pereira, M.F. (2019). A Motivational Case Study in Social Robotics. In H. Ayanoglu \& E. Duarte (Ed.). Emotional Design in HumanRobot Interaction. Theory, Methods and Applications (pp. 9-35). Springer.

Serrano Sánchez, J.L. (2019). Herramientas telemáticas en aulas hospitalarias: Una experiencia educativa en la región de Murcia. https://bit.ly/3hgVR6U

Smith, E.R., Sherrin, S., Fraune, M.R., \& Šabanovi区, S. (2020). Positive Emotions, More Than Anxiety or Other Negative Emotions, Predict Willingness to Interact with Robots. Personality and Social Psychology Bulletin. https://doi. org/10.1177/0146167219900439
Sullivan, A., Elkin, M., \& Bers, M.U. (2015). KIBO robot demo: engaging young children in programming and engineering. Proceedings of the 14 th international conference on interaction design and children (pp. 418-421). ACM. https://doi. org/10.1145/2771839.2771868

Trost, M.J., Ford, A.R., Kysh, L., Gold, J.I., \& Matari冈, M. (2019). Socially Assistive Robots for Helping Pediatric Distress and Pain. The Clinical Journal of Pain, 35(5), 451-458. https://doi. org/10.1097/AJP.0000000000000688

Valencia, N.N, Ortega, J.L., \& Puello, E.C. (2019). Hospital pedagogy: a space of love and recognition for the oncological pediatric patient. Texto \& Contexto Enfermagem, 28, 1-12. http://dx.doi.org/10.1590/1980-265XTCE-2018-0112

Violant, V. (2015). Acción educativa en la infancia en situación de enfermedad y sus implicaciones. En C.G. González y V. Violant (Ed.), Uso de las TIC para la atención educativa, hospitalaria y domiciliaria (pp. 29-43). Mc Graw Hill.

Violant, V., e Molina, M.C. (2010). A formación duns profesionais enquecidos, Como ser mestre ou pedagogo hospitalario? Revista Gallega de educación, 46, 14-16.

Violant, V., Molina, M.C., y Pastor, C. (2009). Pedagogía Hospitalaria. Necesidades, ámbitos y metodología de intervención. Ministerio de Educación de Chile.

Yanow, D. \& Schwartz-Shea, P. (2015). Interpretation and method: Empirical research methods and the interpretive turn. Routledge. 
CARINA S. GONZÁLEZ-GONZÁLEZ, VERÓNICA VIOLANT HOLZ, ALFONSO INFANTE MORO, LORENA CÁCERES GARCÍA, MARÍA DOLORES GUZMÁN FRANCO ROBÓTICA EDUCATIVA EN CONTEXTOS INCLUSIVOS: EL CASO DE LAS AULAS HOSPITALARIAS

\section{Anexo 1}

Proyectos de iniciación de la robótica en hospitales en España

\begin{tabular}{lll}
\hline $\begin{array}{c}\text { Aula } \\
\text { Hospitalaria }\end{array}$ & \multicolumn{1}{c}{ Descripción del proyecto } & URL \\
\hline Aulas Hospitala- & El proyecto consistió en usar la tecnología para & URL: https:// \\
rias Madrid Sur & encontrar soluciones que mejoren su entorno, & www.bq.com/es/ \\
- Centros de Ge- & tal y como facilitar su estancia en el hospital. proyecto-aulas- \\
tafe, Leganés, & Para ello el alumnado con ayuda de los docen- & hospitalarias \\
Móstoles, Alcor- & tes crearon una maqueta automatizada de las & \\
cón y Fuenla- & habitaciones, del aula y añadieron una discoteca \\
brada & $\begin{array}{l}\text { portátil movida por un robot Printbot Evolution. } \\
\text { Además, los niños reprodujeron la sala de juegos }\end{array}$ \\
& $\begin{array}{l}\text { y personalizaron muñecos Playmobil para que } \\
\text { parezcan enfermos. }\end{array}$ \\
\hline
\end{tabular}

Aula hospitalaria El proyecto consistió en introducir la robótica, a de Getafe través del robot Aisoy1 para trabajar los colores básicos, la lateralidad y las expresiones emocionales. Se han realizado dictados, trabalenguas, discriminación auditiva, onomatopeyas, práctica ortográfica, dicción y numeración, con Aisoy1.

URL: https://robotica-educativa. hisparob.es/aisoyen-las-aulas-hospitalarias-de-getafe/

Aula hospitalaria El proyecto denominado "Los robots mejoran mi de Murcia vida", consistió en diferentes fases con secuencias de aprendizajes de acercamiento progresivo a la robótica, con el Kit My Robot y temas STEAM en etapas:

URL:https://robotica-educativa.hisparob.es/ emocionbots-enlas-aulas-hosa) acercamiento a los robots (inclusión de películas, cuentos sobre robots, los robots que nos rodean, las casas del futuro, los drones y máquipitalarias-deleaehd_rm/ nas, etc.);

b) nuestros robots (construcción de un robot colectivo con cajas de cartón, las formas geométricas y los polígonos para hacer robots, construcción de robots de forma individual con materiales constructivos, juegos con los nombres de robots, robots que hablan con frases de robots, los robots que cuentan historias, diálogos de robots, canciones con los robots, historias animadas con robots, etc.);

c) cómo funcionan los robots (programación robótica usando Scratch);

d) creamos robots (sensores, cajas programadas, ejecución de movimientos, construcción de un coche con un motor) y;

e) exposición y difusión de materiales creados (exposición de los materiales creados, contar las historias, difusión por redes sociales). 


\begin{tabular}{lll}
\hline $\begin{array}{l}\text { Aula hospitalaria } \\
\text { de Valladolid }\end{array}$ & El proyecto denominado "Misión a Marte" pro- & URL: http://cfie- \\
& puso la creación de un espacio de colaboración & valladolid.cen- \\
& entre el alumnado ingresado en el hospital y el & tros.educa.jcyl. \\
& alumnado de secundaria del Instituto Cristo Rey & es/bitacora/index. \\
& con el fin de llevar a cabo el aprendizaje de la cgi?wIdPub=1417 & cgíndín y de manera específica en robótica. \\
& tecnología y & \\
& Para publicar las sesiones, materiales para el di- \\
& seño y programación de robots y las actividades \\
& utilizaron para ello foros, blogs (Wordpress), \\
& Youtube y Twitter.
\end{tabular}




\section{PERFIL ACADÉMICO Y PROFESIONAL DE LOS AUTORES} 5939-9544

Carina S. González-González. ORCID: http://orcid.org/0000-0001-

Catedrática de Arquitectura y Tecnología Informática en el Departamento de Ingeniería Informática y de Sistemas de la Universidad de La Laguna (España). Doctora en Informática (2001) por la Universidad de La Laguna y Doctora en Ciencias Sociales y Educación (2020) por la Universidad de Huelva (España). También está certificada en "Early Childhood Technology" por la Universidad de Tufts (EE.UU.). Líneas de investigación: e-learning, gamificación, interacción persona-ordenador, cultura digital. E-mail: cjgonza@ull.edu.es

Verónica Violant Holz. ORCID: http://orcid.org/0000-0003-2464-6845

Profesora titular de la Facultad de Educación. Departamento de Didáctica y Organización Educativa. Universidad de Barcelona. Líneas de investigación: Pedagogia hospitalària, Creativitat, educació i salut y Robòtica educativa. E-mail: vviolant@ub.edu

Alfonso Infante Moro. http://orcid.org/ORCID 0000-0002-0718-7053

Profesor del Departamento de Economía Financiera, Contabilidad y Dirección de Operaciones de la Universidad de Huelva y miembro del Grupo de Investigación GITICE. Doctor en Ciencias Económicas y Empresariales por la Universidad de Huelva. Especializado en Sistemas de Información y Tecnologías, siendo las principales líneas de investigación: TIC-Empresas, TIC-Turismo y TIC-Educación. E-mail: alfonso.infante@decd.uhu.es

Lorena Cáceres García. http://orcid.org/ORCID: 0000-0002-7631-719X

Pedagoga. Exalumna del postgrado de Pedagogía Hospitalaria en Neonatología y Pediatría. Universidad de Barcelona. Líneas de investigación: Pedagogia hospitalària y Robòtica educativa. E-mail: lore28_16@hotmail. com

María Dolores Guzmán Franco. http://orcid.org/ORCID 0000-00021170-3014

Profesora del Departamento de Educación. Sus líneas de docencia e investigación están vinculadas a la integración de TIC en el contexto educativo, la formación del profesorado en espacios educativos virtuales y los estudios en 
competencia mediática. Departmento de Pedagogía, Universidad de Huelva. E-mail: maria.guzman@dedu.uhu.es

Fecha Recepción del Artículo: 16. Marzo. 2020

Fecha Modificación del Artículo: 29. Mayo. 2020

Fecha Aceptación del Artículo: 11. Junio. 2020

Fecha Revisión para Publicación: 23. Junio. 2020 
